\title{
Convergencia mediática: lecciones y preguntas desde la experiencia peruana
}

\section{Media convergence: lessons and questions from the Peruvian experience}

\author{
Eduardo Villanueva-Mansilla ${ }^{1}$ \\ Recibido el 29 de setiembre de 2016 - Aceptado el 28 de noviembre de 2016
}

\begin{abstract}
RESUMEN: La discusión sobre convergencia, representada en variedad de campos académicos relacionados con la comunicación, no ha incluido la producción y el consumo de contenidos, a pesar de la relativa abundancia de referencias a los mismos en documentos empresariales y de política pública en el Perú. Esta situación se aborda proponiendo una definición de convergencia que analiza sus manifestaciones específicas, intentando establecer cómo esta afecta la viabilidad de las industrias mediáticas y la formulación de políticas de comunicación. Se revisa los modelos propuestos para entender la convergencia, y se propone una interpretación que sirva para comprender la convergencia como fenómeno social y cultural.
\end{abstract}

Palabras clave: Convergencia, Industria de Medios, Medios peruanos, Internet en el Perú, Telecomunicaciones en el Perú

ABSTRACT: Discussion on convergence, represented in a variety of academic fields related to communication, has not included production and consumption of content, notwithstanding the relative abundance of references to convergence in Peruvian business and government papers. This is addressed by drafting a definition of convergence analyzing its specific manifestation, trying to generalize how it affects the viability of media industries and the definition of communication policy. Models of convergence are analyzed and an interpretation that helps to understand convergence as a social and cultural phenomenon is proposed.

Keywords: Convergence, Media Industries, Peruvian media, Internet in Peru, Telecommunications in Peru

1 Eduardo Villanueva-Mansilla es Doctor en Ciencia Política y Gobierno, Magíster en Comunicaciones y Licenciado en Bibliotecología y Ciencia de la Información, en todos los casos por la Pontificia Universidad Católica del Perú. Es investigador en temas de Comunicación y Tecnología desde la década de 1990. Es Profesor Asociado del Departamento de Comunicaciones de la Pontificia Universidad Católica del Perú. evillan@pucp.pe, http:// orcid. org/0000-0003-1312-4873. 


\section{Introducción ${ }^{2}$ : ¿’Por qué convergen- cia?}

Convergencia es un término que prevalece en muchas discusiones de política pública, planes de negocios e incluso discusiones académicas, pero que tiene relativamente poca teorización. Sin duda ha sido útil para presentar y fijar un proceso que desde sus inicios en la década de 1990, ha cambiado radicalmente la naturaleza de los medios: el término sirve al mismo tiempo como un sinónimo de disrupción y alteración, y como un descriptor cómodo de algo que sigue ocurriendo y que no sabemos muy bien definir, aunque esté claro que nos afecta a todos.

Aceptando entonces la existencia del término y su referencia a fenómenos específicos, es importante constatar que se lo usa en los estudios sociales de comunicación aunque tampoco se lo haya definido con claridad o precisión. Abundan los aportes, pero no existe consenso sobre a qué nos referimos al hablar de convergencia.

Específicamente para el caso peruano, la cuestión de la convergencia implica plantearse exactamente cómo interpretar un término que es usado, por ejemplo, en documentos oficiales del Ministerio de Transportes y Comunicaciones o del Organismo
Regulador de la Inversión Privada en Telecomunicaciones (OSIPTEL), presentándolo como un proceso o fenómeno fundamentalmente tecnológico, relacionado con el desarrollo de la digitalización de las telecomunicaciones, pero sin mayor consideración de los aspectos sociales y culturales, para no mencionar la relación con las industrias de comunicación social, que implica semejante conjunto de fenómenos.

El origen de la reflexión comunicacional sobre la convergencia considera los aspectos tecnológicos con particular énfasis. Por ejemplo, Garnham (1996) discute un modelo temprano para entender la convergencia basada en la Internet, presentando un número de niveles que describen qué proceso o condición se va desarrollando en la temprana época en la que el artículo fue escrito. En ese entonces existía un conflicto entre las "nuevas telecomunicaciones", basadas en servicios propietarios impulsados por corporaciones, descansando en el modelo de Red Digital de Servicios Integrados promovido por la Unión Internacional de Telecomunicaciones; y el modelo abierto y de libre acceso que encarnaba la Internet (Mansell, 1993). Si bien no era necesariamente obvio que todos los servicios de telecomunicaciones se convertirían en una extensión del protocolo TCP/IP y, en menor medida, del HTTP,

2 El presente texto utiliza partes de un trabajo anterior: Convergence from the periphery: developments in Peruvian media industry and the effects on the production markets, presentado ante el congreso 2016 de la Asociación de Estudios Latinoamericanos (LASA). 
sí era evidente que las soluciones propietarias enfrentaban un escenario muy competitivo frente a la Internet. Las observaciones de Garnham sobre las limitaciones de ingeniería civil a la convergencia pueden ser vistas a la distancia como una prueba de la imposibilidad de prever el enorme impulso que el desarrollo de servicios y mercados basado en TCP/IP significaría para las inversiones en telecomunicaciones, especialmente respecto a la infraestructura portadora tanto fija como móvil, a mediados de los años noventa.

Incluso ahora, cuando conglomerados de contenidos y servicios audiovisuales plantean campos de batalla entre las empresas tradicionales de medios, las transnacionales de telecomunicaciones, y los gigantes computacionales de consumo final agrupados bajo el acrónimo GAFA ${ }^{3}$, es la Internet la red que impulsa la convergencia, y son los sobrevivientes, como la televisión de señal abierta, los que son vistos como próximas víctimas de la inminente migración a las redes IP. Tan solo la radio se mantiene, dado que las estaciones digitales no han logrado masificarse, y la mayor parte del consumo de estos servicios sigue patrones tradicionales aunque el contenido haya cambiado, por ejemplo al abandonar la industria de la música a la radio como mecanismo de difusión de nuevos productos.
También es cierto que "convergencia", en el más vago de los sentidos, es usado por conferencistas profesionales, consultores e incluso académicos para indicar una situación en la que los nuevos desarrollos tecnológicos o mediáticos están transformando arreglos tradicionales en muchas industrias, mercados y prácticas de consumo. Esto apunta a la convergencia como un constructo, un concepto construido a partir de la observación de fenómenos sociales varios que no agrupa ni discrimina sistemáticamente las distintas posibilidades explicativas que invocaría. Entonces, las definiciones propuestas son hasta cierto punto cambiantes: describe fenómenos y epifenómenos pero no explica de donde provienen ni hacia donde van.

Poder entender la situación en el Perú requiere entonces aceptar tanto la mirada estrictamente tecnológica, predominante en documentos oficiales, presentaciones de funcionarios y opiniones de consultores; así como desde una perspectiva académica que considere la experiencia de la comunicación social, en donde los conceptos han ido desarrollando de otra manera. Por ello, se comenzará por la discusión conceptual, para luego pasar a la situación en el Perú más allá de las telecomunicaciones, lo que nos permitirá establecer la pertinencia y características de los distintos conceptos de convergencia para el país.

3 La combinación de Google, Apple, Facebook y Amazon, los actores dominantes en servicios globales de comunicación basada en redes IP. 


\section{Modelos conceptuales}

Una mención más contemporánea proviene de Becerra (2015), para quien "infocomunicación" indica la novedosa combinación de medios y telecomunicaciones, con la convergencia como el proceso del que resulta dicha combinación. En un espíritu similar, el proceso convergente es destacado por Jenkins (2006) como el productor de una forma cultural novedosa, la llamada "cultura de convergencia", creando un andamiaje teórico para sostener el proceso. $\mathrm{Al}$ menos en dos ocasiones, pero de manera general, Jenkins propone definiciones operacionales de convergencia, fundamentalmente el flujo de contenido a través de múltiples plataformas, lo que es muy cercano a las definiciones habituales de transmedialidad; pero también indica la cooperación entre muchas industrias mediáticas, lo que se acerca a una forma tecnológica de convergencia expresada en servicios manejados por una empresa o varias en alianza; y finalmente lo que llama "el comportamiento migratorio" de los consumidores, que irán a donde sea necesario para encontrar el contenido deseado (Jenkins, 2006: 2-3). El autor acepta que "convergencia" no quiere decir algo específico, sino algo específico para el que lo dice, desde su propio campo o actividad.

Podríamos enfrentar esta situación partiendo del aserto de Jenkins que el uso diverso del término por muchos profesionales de distintas disciplinas indica que de existir, la convergencia es un fe- nómeno multifacético que debería ser analizado desde distintos marcos explicativos. Además, se puede recomendar que de usar convergencia, debería indicarse explícitamente a qué dimensión o faceta de la misma se está haciendo referencia. Finalmente, el punto de partida debería ser también explícito: ¿nos preocupa que distintos actores estén confrontados en el mercado como agentes económicos? ¿̇o es una cuestión de arreglos institucionales que varían de un estado nación a otro? co hablamos de la gente y cómo cambia su relación con los medios? En otras palabras, ¿cuál es el sujeto de la convergencia?

El otro factor definitivo asociado a la convergencia es su naturaleza global. Dado que el impulso que la provoca es la Internet, entonces los efectos de la expansión de acceso y uso de la Internet, al ser globales pero asimétricos, despliegan convergencia global y asimétricamente. El punto de partida debería ser entonces la penetración de la Internet y sus efectos en los distintos terrenos en donde podemos postular que hay procesos convergentes.

A partir de esto, podemos proponer una definición operacional para continuar el análisis. Entenderemos convergencia como un proceso en el que dos o más elementos parte de un conjunto comunicacional, se unifican en un nuevo elemento, o son subsumidos en un elemento dominante que captura sus funciones hasta transformarse él mismo en algo nuevo. La variante contemporánea enfatizaría que el vector primor- 
dial del proceso de unificación o subsunción es la Internet.

Visto así, un proceso convergente típico sería lo ocurrido con los teléfonos móviles. Originalmente dispositivos que servían exclusivamente para realizar llamadas o enviar mensajes, poco a poco fueron incorporando funcionalidad propia de otros dispositivos portátiles, hasta que el smartphone, en la forma del primer iPhone en 2007, permitió unificar todas las distintas funcionalidades en una sola plataforma, que ya no se usa predominantemente para ejercer su función original (llamadas telefónicas) sino para servicios emergentes que han aprovechado la combinación novedosa de funciones para crear nuevas posibilidades comunicacionales. El smartphone es un ejemplo ideal de convergencia de dispositivos, pero que también ha colaborado para lograr convergencia de servicios y de industrias.

El ejemplo de los smartphones sirve para reforzar la idea que los procesos convergentes tienden a ser globales, debido al grado de integración transnacional de los operadores de telecomunicaciones, proveedores de dispositivos y de contenido, y experiencias de comunicación, que existe en el período de la comunicación por Internet. Es natural que una red de alcance global y uso transparente como la Internet cree condiciones para que las tendencias de la globalización económica se reflejen en la vida cotidiana de las personas, a través del consumo mediático; la tecnología facilita la personalización de la ex- periencia pero depende de la provisión de servicios globales, y en este sentido solo podemos hablar de convergencia tomando en cuenta el impulso de la globalización y la liberalización del comercio internacional, incluso en los casos en que los sistemas políticos ponen barreras al acceso (como en países autoritarios como China).

Postulando que la convergencia es multivariable y multifacetada, podemos presentar al menos cinco tipos de convergencia, recogiendo las diversas fuentes en donde se discute el término.

a. Convergencia tecnológica: considerando la conversión de la mayoría de servicios de comunicación a redes basadas en el protocolo TCP/IP, más el surgimiento de servicios como conectividad IP en los hogares y en suscripciones móviles, es posible analizar y discutir un tipo específico de convergencia en la cual los principales actores son empresas de telecomunicaciones, y donde el conjunto comunicacional en proceso de fusión es la suma de servicios individuales, como la telefonía de voz, el fax y los servicios portadores, que están siendo subsumidos en plataformas IP que permiten, mediante adaptaciones de software, proveer símiles de los servicios originales, pero sin las limitaciones preexistentes (Crandall y Waterman, 1995; Finn, 1999, son análisis tempranos de estos fenómenos); la gran diferencia es que no son las empresas de telecomunicaciones las que definen 
y brindan el servicio final, como ocurriría en los tiempos de la telefonía de voz, por ejemplo; ahora las redes simplemente sirven para transportar datos que son convertidos en comunicación por servicios bajo el control de otros actores, como el caso de los servicios de conversación que ahora, lentamente incorporan comunicación de voz de escala global. Esta visión integradora fue avizorada desde la década de 1970 (Farber y Baran, 1977), pero no contaba con las facilidades técnicas para llevarse a cabo. Un aspecto que también destaca en estas formas del proceso es la llamada "convergencia de dispositivos", como se discute en Villanueva-Mansilla (2000) y en Ishii (2006). Otras miradas incluyen la discusión sobre convergencia tecnológica y su relación con la expansión de los servicios móviles (Humphreys, Von Pape y Karnowski, 2013)

b. Convergencia de servicios: con énfasis en el consumo de servicios y contenidos, y en los efectos de dicho consumo en la formación y desarrollo de audiencias tanto a nivel local, nacional, regional e internacional. Desde servicios de mensajería o comunicación interpersonal, como WhatsApp, Skype o Telegram, que han transformado nuestra forma de comunicarnos por voz o texto; hasta el contenido disponible por stream en audio (como Spotify) o video (como Netflix), lo que predomina en la actualidad es un conjunto de actores transnacionales que no son regulados localmente aunque hagan uso de recursos bajo la égida del estado, o así no cumplan con estándares de contenido de aplicación nacional. En el proceso, estos servicios desbaratan viejas audiencias, estructuradas nacional y localmente por los medios masivos de comunicación de antaño bajo reglas que cambiaban de país en país. Al hacerlo, estos servicios transnacionales crean nuevas experiencias y oportunidades, las que a su vez crean nuevas audiencias de naturaleza distinta: el corte no está articulado por el estado-nación, sino por las características socio económicas de grupos diferenciados por su actitud cosmopolita o localista (Hier, 2008; Melody, 2014). Esto también puede incluir lo que Garnham (1996: 106) llamó "convergencia de modos de pago", algo mucho más complejo que aún no se ha expandido fuera de los confines de ciertos estados-nación particularmente inclinados a la virtualización de operaciones financieras a nivel del retail. Además, la dimensión empresarial es fundamental, dado que son solo aquellos conglomerados capaces de acceder y usar cantidades elevadas de capital financiero las que pueden operar en los mercados de servicios convergentes (Gutiérrez-Rentería y López, 2014). McChesney (1999) consideró que la convergencia era simplemente la posibilidad que las industrias mediaticas y las de telecomunicaciones compitieran tanto entre sí por nuevos mercados, como por la invasión de los mercados de una y otra. 
c. Convergencia regulatoria: tomando como punto de partida el prisma legal, se debaten los muchos aspectos regulatorios que incluye la integración de mercados en Europa; privacidad y protección de datos; concentración de propiedad y protección a los consumidores; alianzas globales y efectos locales, etcétera (para mayor discusión, ver Mastrini y Bolaño, 1999; Neuchterlein y Weiser, 2005; Santander 2014; Tambini, Leonardi y Marsden, 2008; Valtysson, 2012; Weinstein, 2004; entre otros).

d. Convergencia de contenidos / convergencia cultural: basándonos en aproximaciones como la propuesta por Jenkins, una cultura de convergencia estaría basada en nuevas prácticas culturales construidas a partir de las posibilidades que emergen de las prácticas convergentes construidas desde los medios e industrias de la comunicación. Ciertamente, esta aproximación también se refiere a un conjunto significativo de industrias culturales, que como se mencionó antes, no dependen de portadores específicos o de tecnologías de entrega de contenidos (como la televisión, sea de señal abierta o cable), y que no requieren de la estructura de mercado propia de las industrias mediáticas masivas. Esto ofrece una evidente conexión con la convergencia de servicios, aunque el foco de atención yace en los efectos culturales y la relación con las formas narrativas y expresivas en general que nacen en la producción y el consumo de estos contenidos. Jenkins (con Ford y Green, 2013) propone el concepto de spreadable media ${ }^{4}$, afirmando que el involucramiento de los fans en la re-creación y circulación del contenido mediático no es tan solo un efecto secundario de interés, sino una fuerza significativa de empoderamiento de dichos aficionados, así como también un posible camino para la explotación comercial de esos recursos. Scolari (2015) establece paralelos entre las hipermediaciones y la tradición de estudios de mediaciones; McPherson (2008) indica que las interfaces, es decir los mecanismos a través de los que se interactúa con el contenido, transforman nuestra relación con el mismo, y consecuentemente nuestra expectativa de intercambio y consumo cultural. Por su lado, Webster y Ksiazek (2012) exploran la transformación de las audiencias a través del consumo simultáneo de televisión y video en stream, las llamadas experiencia de segunda pantalla, para indicar que el contenido atrae dedicación comparable sin importar la naturaleza del medio técnico usado, lo que apunta a la debilidad fundamental de los medios frente a la

4 Es casi intraducible, pero podría decirse que se refiere a medios que se untan sobre las superficies culturales y sociales con mucha facilidad. "Spread" es un término común para alimentos que se pueden untar, pero resulta muy poco intuitivo llamarlos "medios untables" en español, por lo que quedará en inglés. 
fuerza del contenido, haciendo evidente que no habrá una limitación cultural de fondo al abandono de la experiencia televisiva tradicional en favor del consumo por redes IP, cuando la maduración de los productos alcance el nivel necesario.

e. Convergencia expresiva / profesional: alterando la perspectiva, esta mirada ofrece un acercamiento a las consecuencias de la convergencia desde los profesionales de la comunicación, que tienen que lidiar constantemente con la realidad de muchos medios que son unidos por nuevos actores que intentan salvar las viejas fronteras entre formatos convencionales hacia nuevas formas, muchas veces experimentales, de storytelling, incluyendo lo conocido como transmedialidad (Scolari e Ibrus, 2014). Los nuevos arreglos que afectan al periodismo para alcanzar máxima eficiencia y cobertura pueden ser entendidos como parte de esta comprensión específica de la convergencia (Salaverría 2008, entre otros), un debate bastante bien documentado sobre los efectos profesionales en el periodismo como profesión. (Cooke, 2005; Lim, 2010; Mitchelstein y Boczkowski, 2013; Robinson, 2011; Russell et al, 2008). Otros términos, como Post$\mathrm{TV}$, o "convergencia internet-televisión” (Campbell, 2011), remiten tanto a una convergencia del tipo de servicios, como a las innovaciones expresivas que toman las oportunidades creadas por la Internet como pretexto para explorar nuevas formas de contar historias, mediante experiencias que a veces pueden ser anatemas para los dedicados a un medio expresivo específico como el cine, como lo discute Beugnet (2013). En el caso del periodismo, las transformaciones son evidentes en nuevas formas de organización profesional (Salaverría, 2008), en el consumo de noticias a través de múltiples plataformas (Yuan, 2011), y en la presencia del tema en las discusiones periodísticas (Lin, 2013).

Ciertamente, es posible proponer otras miradas, pero en este listado particular el énfasis yace en la identificación de los actores dominantes que impulsan cada aproximación analítica específica de la convergencia. Por ejemplo, si decidimos que lo más significativo de la convergencia es su dimensión técnica, entonces las corporaciones transnacionales que dominan tanto las telecomunicaciones como los servicios por computadora y el hardware, serán los actores críticos a considerar, con mayor peso que un gobierno nacional o una nación económicamente emergente. Por otro lado, la convergencia expresiva implica a actores comerciales en mercados específicos, en los cuales es posible resistir la andanada de demandas técnicas a partir de creatividad aplicada, o simplemente aprovechando el potencial de nuevos medios para escapar de las limitaciones comerciales que los viejos medios imponen.

Regresando al Perú, la convergencia resultará no solo de estas tendencias 
globales, sino de una serie de filtros específicos a la experiencia nacional: penetración de la Internet, en comparación con la penetración de otros servicios mediáticos; patrones de uso de la Internet frente a aquellos de otros servicios de contenido; provisión de contenidos, como reflejo de patrones transnacionales pero frente a los patrones nacionales de los medios tradicionales; y factores complementarios, como la apertura de la economía; tarifas y costos de uso e implementación de servicios mediáticos; y tradiciones culturales en relación con la producción de contenidos mediáticos, a todos los niveles posibles, desde la radio y prensa local hasta contenido digital exportable.

\section{Las particularidades de los medios peruanos}

El incremento en la producción cinematográfica originada en Lima ha sido, en los últimos años, visible. La amplia variedad de temáticas y calidades de las películas estrenadas desde 2010 en adelante refleja mayor capacidad de consumo e interés de parte de los consumidores. El éxito específico de ciertas producciones augura que la producción local continuará por varios años.

Sin mayor conexión con este ciclo de producción, e incluso previo a él, el fenómeno paralelo del "cine regional" no ha tenido mayor impacto en las salas limeñas, la ciudad donde se concentra la mayoría de la riqueza del país. Definido como producciones en ciudades inter- medias, sobre todo en la zona andina, con medios modestos, basados en narrativas locales, este cine ha tratado sobre todo de tropos de horror / terror, con un grado de éxito llamativo, pero fuera de los patrones tradicionales de distribución, recurriendo a la distribución de copias hechas individualmente y la comunicación interpersonal (Bustamante y Luna Victoria, 2014). Películas como "Qarqacha, el demonio del incesto" y "Incesto en los Andes: la maldición de los jarjachas", ambas de 2002, fueron hechas con bajos presupuestos y lograron ventas importantes a pesar de que no llegaron a salas formales en Lima, hasta el punto que continúan siendo básicamente desconocidas por las audiencias de la capital. Un director en particular dijo en 2015 que su película más barata fue hecha con un gasto de 100 soles, no más de 40 USD al cambio del momento (Gallegos, 2015).

Este ejemplo muestra la distancia que separa la producción mediática peruana: la esfera nacional, centrada en Lima, gira alrededor de audiencias definidas por gustos y capacidades de consumo de un centro urbano con una clase media relativamente grande; en las "provincias", donde reside una cantidad mucho menor de aquellos ciudadanos con capacidad de consumo y gustos comparables a los "limeños", el contenido nacional puede estar disponible, pero existen grupos artesanales con buena salud aunque con recursos escasos que abastece el consumo y los gustos locales. Este patrón es más significativo en las zonas andinas, donde la conexión con lo li- 
meño es culturalmente más tenue (Morales et al., 2009).

La industria de los medios en el Perú tiene una alta concentración geográfica en Lima, la capital del país. Dado que aproximadamente un tercio de la población y cerca del $70 \%$ de la economía está basada en el área metropolitana de la capital, no es realmente sorprendente. Todos los diarios, cadenas de radio y televisión, públicas o privadas, están basadas en Lima, así como las estaciones de televisión de pago de alcance nacional; los sitios web, incluyendo blogs populares y servicios de noticias. Si bien existen diarios locales de importancia en ciudades además de Lima, su alcance está limitado a su área de origen (Mendoza, 2015), al igual que algunas estaciones de radio y televisión; además adolecen de escasez de recursos, tanto profesionales como técnicos.

Para todos los segmentos mediáticos, el capital de origen peruano predomina, aunque con niveles significativos de concentración de propiedad: el grupo El Comercio tiene aproximadamente $70 \%$ del mercado de consumo de diarios (Huamán y Becerra, 2014); dos cadenas, con una variedad de estaciones especializadas en distintas audiencias, dominan el mercado nacional de radio; cuatro cadenas privadas y una estatal controlan el grueso del mercado televisivo de señal abierta, con varias señales digitales toda- vía buscando audiencia y sin fecha clara para un apagón analítico incluso en la capital. Una de las cadenas es controlada indirectamente por capitales mexicanos (Angel González, conocido como El fantasma, quien controla cadenas de televisión en varios países latinoamericanos)

Una vez que se dejan los medios masivos tradicionales, el control cambia de manos: la televisión paga, los servicios de telecomunicaciones y de telefonía móvil, y el acceso a Internet, es controlado por conglomerados transnacionales. Movistar, parte del Grupo Telefónica de España, domina claramente el sector de televisión de pago ${ }^{5}$, con 1.114.119 suscriptores a diciembre de 2015; tanto América Móvil (México) como DirecTV (USA), tienen cantidades menores de suscriptores, alrededor de 216.000; el cuarto más grande, apenas 25.000 suscriptores, y el resto, compuesto por unos 200 operadores a nivel nacional, 106.000 usuarios (Cassano, Dettleff y Vasquez, 2015).

Los servicios de telecomunicaciones, incluyendo el acceso a la Internet, son fundamentalmente un duopolio, controlado por dos grandes multinacionales, Telefonica / Movistar y América Comunicaciones / Claro, con competencia limitada en el campo móvil y algunos operadores especializados. A su vez, la mayor parte del consumo de Internet tiene los mismos operadores.

5 Todas las cifras sobre televisión de pago provienen del regulador, OSIPTEL, https://www.osiptel.gob.pe/ articulo/74-suscriptores-por-empresa-a-nivel-nacional-mensual. 
Este acceso es, salvo para la radio, mayor en Lima que en el resto del país ${ }^{6}$.

Esta concentración de servicios de telecomunicaciones marca una gran diferencia con realidades en la región, como Argentina, donde grupos empresariales locales e internacionales compiten en varias arenas (Becerra, 2015). En el caso peruano, prácticamente existe un equilibrio homeostático entre los grandes operadores, y no hay capitales locales en condiciones de ingresar a competir en asuntos de telecomunicaciones. Esta situación se agudiza por el hecho que la privatización de telecomunicaciones de la década de 1990 permitió que Telefónica comprara la infraestructura completa existente, dándole control vertical del sector, y creando una barrera de acceso significativa a cualquier competidor al no exigirle compartir infraestructura; el resultado es que competir con este operador requiere inversiones significativas que no resultan viables en mercados consolidados como las telecomunicaciones; el nuevo plan de banda ancha (Perú-MTC, 2011) busca que aparezcan nuevos operadores mediante el alquiler de una red ya disponible, pero es un pendiente dado que la red todavía no está lista (en setiembre 2016).

Tabla 1: Acceso a servicios, porcentaje de hogares

\begin{tabular}{|l|r|r|}
\hline Servicio & Lima & Provincias \\
\hline Radio & 79,9 & 78,3 \\
\hline Televisión & 97 & 76,2 \\
\hline Televisión de pago & 61,4 & 24,5 \\
\hline Internet & 41,5 & 14,6 \\
\hline
\end{tabular}

Fuente: INEI. Elaboración propia

Tomando en cuenta tanto el ejemplo inicial como las cifras aquí expuestas, resulta evidente que en el Perú hay dos mercados de contenidos: uno basado en Lima, dominado por actores de alcance nacional con distinta segmentación de contenidos y distintas audiencias que satisfacer, y el atendido por los dispersos y variados mercados regiona- les, donde la producción es de tipo más artesanal (Dettleff, 2012).

Otra dimensión emergente es la comunicación multiplataforma, entendida como transmisión simultánea en medios tradicionales y servicios IP, sean fijos o móviles. Predominante en las radios, las cadenas televisivas hacen menor uso del

6 La información sobre penetración de servicios proviene de las estadísticas oficiales más recientes del Instituto Nacional de Estadística e Informática, (https://www.inei.gob.pe/estadisticas/indice-tematico/tecnologias-de-lainformacion-y-telecomunicaciones/). Estas estadísticas no son las mismas a las estimadas por los estudios de ConcorTV, que usan diseños muestrales para calcular la penetración de servicios (http://www.concortv.gob.pe/ investigacion/estudios-cuantitativos/2016-estadisticas-de-la-radio-y-television-en-el-peru/). 
mismo, al menos en el sentido estricto, con más uploading de contenidos a YouTube que con plataformas propias de retransmisión. El contenido digital resulta complementario antes que central, y es más un recurso promocional que una ruta de monetización de contenidos. El contenido digital, como series web, juegos y similares no tiene rutas claras de monetización aunque pueda ser relativamente abundante. Algunos intentos han sido o siguen siendo exitosos en sus segmentos específicos de mercado, como por ejemplo Los Cinéfilos, muy popular serie web sobre aficionados al cine, tiene seis temporadas con 64 episodios de cuatro minutos de duración; el éxito se refleja en la producción de películas para cine basadas en personajes distintos pero por los mismos intérpretes.

Nuevamente, la mayoría del contenido para los medios digitales se produce en Lima, para los estándares asumidos de clase media que definen el estilo cultural predominante, con elementos que pueden reconocerse como culturalmente "populares", de manera gruesa o estereotipada. Las manifestaciones culturales más allá de la clase media limeña suelen estar disponibles en las horas o señales de menor audiencia, en los medios masivos; y en sitios web dirigidos a grupos de fuera de la capital o de menores ingresos en general. Mientras tanto, los usuarios con mayor conexión acceden a las riquezas de la cultura global de consumo, pudiendo así ignorar las ofertas locales en búsqueda de satisfacer vocaciones cosmopolitas de inserción y participación cultural. Desde la música al video especializado en géneros como anime y cine de la India, así como el gaming o juegos de videos, la Internet es la puerta para una abundancia que no estará disponible en la televisión de pago. Esta minoría, que no está limitada a los sectores de mayores ingresos, hace también uso de recursos informales o irregulares para el consumo (como la piratería, los servicios para compartir archivos, torrents y demás), con pocas limitaciones efectivas desde el control legal de dichos servicios.

El contraste entre concentración a nivel nacional con los servicios locales es llamativo. Las ciudades intermedias, entre 100.000 y un millón de habitantes, tienden a contar con medios locales bien establecidos, con diarios, estaciones de radio y televisión claramente operando como negocios distintos; algunos de los diarios o revistas tienen ocasionalmente presencia nacional al tratar temas locales de importancia general. Sin embargo, en los extremos, como las áreas urbanas llamadas "zonas emergentes" pero que se conocían como barriadas o zonas marginales hasta hace un tiempo, las estaciones de radio e incluso televisión son negocios precarios, funcionando desde residencias particulares, con gran escasez de recursos. En ciudades más pequeñas, hay medios "convergentes", en el sentido que la misma familia maneja en el mismo hogar prensa, radio, televisión e Internet con equipos aprovechados para cada actividad según sea necesario, y con producción muy barata de los bienes finales. 
El mercado de la música, en cambio, refleja con más claridad que antes la existencia de los dos grupos de audiencia ya mencionados. El colapso global de la industria de la música grabada significó el abandono por los conglomerados locales de la escena local, siendo reemplazada lentamente por servicios de streaming y venta de versiones digitales; pero en el camino la informalidad, particularmente barata en la música, se hizo cargo de las necesidades de consumo. En la actualidad, los músicos "limeños" han creado un mercado de conciertos y grabaciones informales similar al que describe Tucker (2013) para el huayno: empresas que montan negocios con costos bajos y mecanismos de venta oportunista.

Este recorrido sumario por algunas de las industrias culturales y de telecomunicaciones del Perú ha mencionado muy poco el término "convergencia". Esto apunta a la ausencia de estrategias comerciales que puedan realmente llamarse tales, dado que el mercado se haya concentrado en pocos actores (y la concentración no implica convergencia si no se ofrecen los servicios indicados); las experiencias multiplataformas son derivativas, casi casuales; y la fisura o clivaje entre los mercados cosmopolita limeño y localista del resto del país crea una separación compleja para aprovechar el potencial adquisitivo del total de los consumidores en ruta a una industria convergente local. El uso intenso de medios sociales como Facebook indica que las conexiones disponibles son usadas con fines que podríamos llamar "cosmopolitas": expresión y comunicación similar a la que predomina en los países del norte global. Con cinco países entre los que tienen una mayor penetración de uso per capita en el mundo (CEPAL, 2015: 63), lo que predomina una vez que se cuenta con acceso no es la búsqueda de contenido local o nacional, sino global, incluso en países gigantes en la producción de contenido (CEPAL, 2015: 64).

En cuanto a las políticas públicas, el tema tiene presencia en lo tecnológico pero no en lo comunicacional: el plan nacional de banda ancha (Perú-MTC, 2011), el documento oficial más reciente sobre TIC / Telecomunicaciones, considera a la convergencia como un tema de tecnología, tanto al nivel de red y dispositivos; tiene algunas consecuencias en la provisión de contenidos, como parte de un circulo virtuoso que puede estar sucediendo, pero no define ni comenta los resultados de dicha convergencia fuera del ámbito de las telecomunicaciones. Se la ve como una oportunidad para otras industrias, que podrían usar este entorno habilitante para ampliar sus iniciativas y buscar, a través de nuevas inversiones, ampliar o crear mercados. Esta actitud es consistente con las políticas de inversión y desarrollo económico que el gobierno peruano ha sostenido desde 1990, apoyándose en actores privados en mercados abiertos.

Por el lado de las industrias culturales, el Ministerio de Cultura del Perú (www. cultura.gob.pe) tiene una Dirección del 
Audiovisual, la Fonografía y los Nuevos Medios, dedicada sobre todo a la producción y promoción cinematográfica. No hay menciones en este sitio a la producción alternativa o no convencional de contenido, el uso de variadas plataformas, o convergencia.

Para terminar, el acceso a los servicios que brindan las telecomunicaciones varía no solo por ingresos, sino por la penetración y calidad de las redes. La mayoría de las conexiones de banda ancha se ubican en las zonas costeras del país; las regiones andinas y selvática cuentan con mucho menor velocidad, por razones técnicas y comerciales. Los consumidores sin acceso directo / domiciliario a la Internet han usado por mucho tiempo las "cabinas públicas", el nombre peruano para lo que se suele llamar "cibercafé" (Averweg y Villanueva-Mansilla, 2009). En el Perú, estas cabinas suelen persistir en las zonas de bajos ingresos, además de las áreas comerciales en donde el público en general puede querer acceder a servicios específicos. A inicios de la década pasada las cabinas eran el modo preferido de conexión, pero esto ha cambiado y las que continúan funcionando en Lima tienden a estar orientadas a video juegos.

Todo esto refuerza la noción de un mercado de dos pisos, donde algunos consumidores tienen a su alcance mucho más contenido, mientras que otros solo tienen la oferta de origen local para medios, contenidos e incluso tecnología en el amplio sentido del término. Los habitantes de Lima cuentan con medios transnacionales y nacionales para abastecerse de contenido local, regional y global, aunque el determinante final de acceso no será exclusivamente la ciudad en la que se vive: hay variables económicas y también culturales que llevan en una $\mathrm{u}$ otra dirección (VillanuevaMansilla, Nakano y Evaristo, 2015). Pero cuando se sale de Lima, las posibilidades se van reduciendo hasta centrarse casi por completo en lo local.

¿Cómo pensar en la convergencia bajo este contexto? Un joven que vive en una ciudad pequeña del Perú puede gozar de convergencia tecnológica, en el sentido de tener acceso a la Internet de banda ancha, incluso a través de un dispositivo móvil. Él o ella pueden tener acceso a servicios convergentes; pero la dislocación entre el contenido localmente relevante y contenidos atractivos de origen nacional o internacional trae la pregunta sobre la pertinencia de hablar de convergencia sin considerar la dimensión sociocultural y finalmente, comunicacional, en un país como el Perú.

\section{Un modelo para comprender la con- vergencia: considerar las dimensiones socioculturales}

La convergencia ha sido usada como una forma de comprender una serie de condiciones que han ocurrido por la acción o inacción de una variedad de actores públicos y privados, en muchos campos relacionados con los medios y las telecomunicaciones, fundamental- 
mente por la influencia de la Internet como red capaz de subsumir en ella todas las formas de comunicación habidas y por haber. Analíticamente, es una aproximación bastante gruesa, que comienza a tener sentido cuando se la entiende como afacetada o de múltiples capas, en vez de un proceso o estado único. Pero su poder explicativo yace en una premisa que no es necesariamente relevante para todos los países y jurisdicciones en donde los síntomas de la convergencia puedan ser detectados: que algo así como un proceso convergente está ocurriendo.

Dicho de otro modo: las transformaciones tecnológicas impulsadas por el éxito de la Internet como un medio de propósito general para la transmisión de señales, tienen lugar en todas partes, como resultado de la adopción general de la Internet por las industrias de telecomunicaciones y también de medios. Además, un número de servicios, gracias a dicha opción tecnológica, comienzan a configurar un mercado global de oferta, con variantes nacionales por cuestiones legales y regulatorias; este mercado global compite por la atención de los consumidores con los mercados nacionales en varios niveles, desde el comercial hasta el cultural. El entorno habilitante se crea a través de tratados, leyes y normas de distinto nivel fomenta el despliegue tecnológico, pero una vez establecido genera presión para que se faciliten los servicios que aprovechan estos entornos, lo que a su vez crea presión para que la circulación de bienes culturales sea también facilitada.
Finalmente, en los mercados más competitivos, distintos grados de convergencia profesional o expresiva, forzada por los actores transnacionales que irrumpen localmente, fuerza su espacio en las industrias mediáticas tradicionales, como la prensa o la televisión.

Esta descripción incluye necesariamente una dimensión global: se asume que la convergencia, en sus distintas formas, irradia de los centros tecnológicos y de medios del mundo hasta inevitablemente, cubrir el planeta entero con un nuevo orden industrial, dominado por los operadores convergentes como GAFA o los unicornios (Harvard, 2016), que incluyen actividades no mediáticas como el transporte personal, encarnado entre otros por Uber. Esta dinámica tendrá lugar en todos los países según sea viable y conveniente para todos los interesados, y algunas firmas locales podrán resistir a través de alianzas o innovación, pero al final tendremos un gran mercado global.

Pero incluso si se acepta que la convergencia como ha sido descrita, arrasará con todo y creará ese mercado global, este escenario específico tiene que ponderar las consecuencias específicas de la convergencia desde la perspectiva de cada sociedad y sus intereses nacionales, no solo desde el comercio sino también desde la cultura, la cohesión social y el debate público. McChesney planteaba (1999: 123) que la consecuencia más clara de la convergencia será una ola sin precedentes de fusiones y alianzas de firmas de medios, telecomunicaciones y 
computación: diecisiete años después, la existencia de lo que llamamos GAFA es precisamente la prueba que ese escenario era válido, y que el dominio de relativamente pocas empresas globales sobre lo que en el fondo siempre tendrá una dimensión local es un tema delicado, dado el costo sobre diversidad y control local que implica.

Desde esta perspectiva, la presión comercial sobre los consumidores llevaría a la homogeneización del consumo, lo que disminuiría el potencial de diversidad local. Esto se debería a la consolidación de los actores industriales alrededor de pocas firmas globales. La penetración de la convergencia tecnológica primero, y luego la de servicios, crea vectores de globalización, que ofrecen similares prácticas y objetos de consumo a aquellos en condiciones económicas y culturales de aprovecharlos.

Bajo esta premisa, si bien la convergencia tecnológica puede penetrar muchas capas de ingresos en una sociedad dada, la convergencia de servicios tiene limitaciones debido tanto a la provisión de servicios competitivos (la capacidad de pago de los consumidores y la posibilidad del mercado en su conjunto de ser atractivo al proveedor). Pero lo más interesante sería la convergencia cultural, más allá de los aspectos profesionales pero incluyéndolos, pues requiere sensibilidad cultural, la que no siempre está asociada con capacidad de pago. Por ejemplo, sectores de la población con capacidad de consumo (personas con mayores ingresos como cabezas de familia) no siempre estarán atraídos por la oferta convergente; pero para los que sí lo están pero no cuentan con los medios, se crea un incentivo para buscar rutas alternativas de consumo (como la piratería).

En otras palabras, muchos países del mundo han sido afectados por la convergencia, aunque no se haya originado en sus economías o por sus propias industrias. Lo que se define como beneficios para los consumidores variará de acuerdo a las posibilidades de cada consumidor, creando asimetrías de consumo que lleva a desequilibrios comunicacionales, entendiendo comunicación más allá de las facilidades técnicas o la oferta comercial.

Para conceptualizar este proceso, el concepto de kludge propuesto por MacKenzie (2010) es especialmente útil. Kludge, según el diccionario Merriam-Webster, es una colección desordenada de partes reunidas para cumplir un propósito particular; una improvisación que cumple un propósito sin estar propiamente bien hecha. Así, la convergencia no es un proceso claramente diseñado para alcanzar un propósito sofisticado o colectivo, sino que es algo que resulta de usar lo que se tiene (digamos, redes y dispositivos TCP/ IP) para lograr el fin buscado, pero donde cada actor improvisa su propia respuesta con los mismos medios. La convergencia, sigue MacKenzie (2010: 99), sería una reducción a un conjunto bien definido de asuntos, pero un kludge implicaría "relacionidad", es decir 
Imagen 1. Penetración de la convergencia

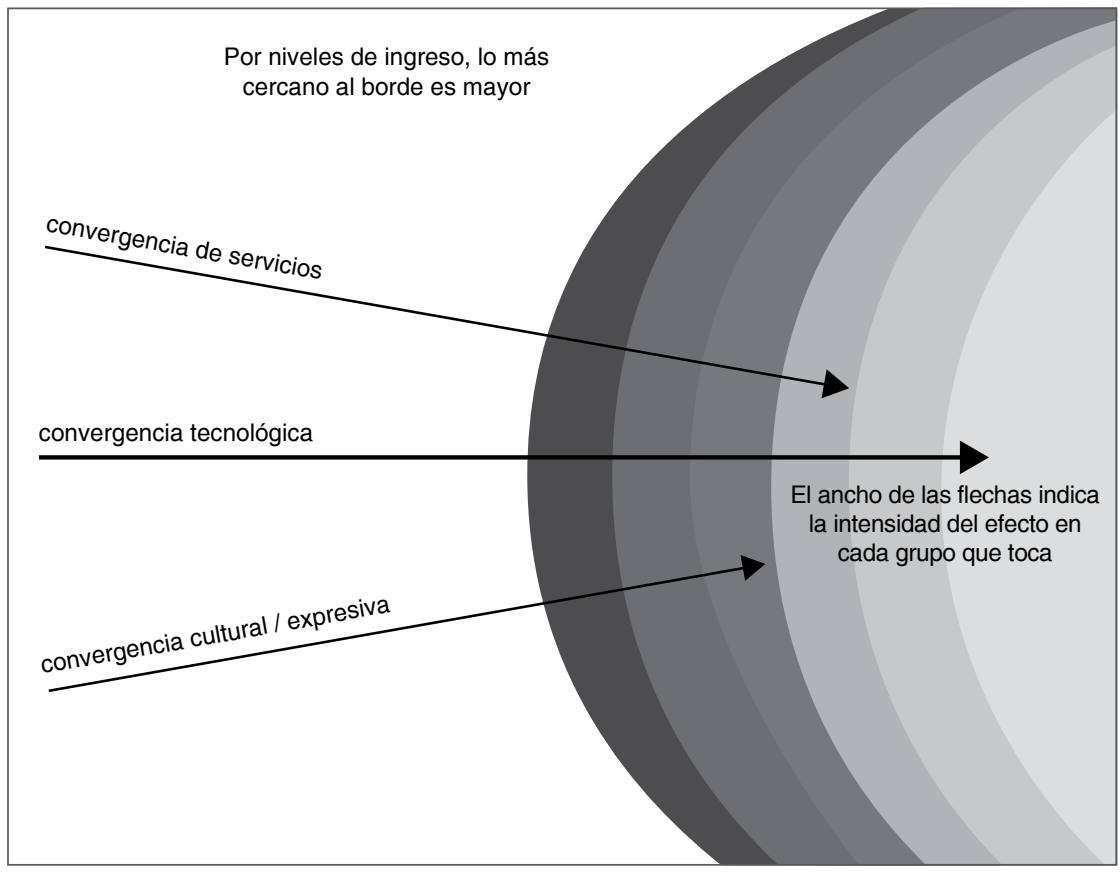

Elaboración propia

una tendencia a establecer relaciones entre elementos que producen cambios que surgen de yuxtaposiciones. Si aceptamos esta mirada, la convergencia es el resultado, no el proceso, dado que el telos se redefine conforme se buscan resultados precisos usando las partes disponibles.

Sería la longitud y fortaleza de dichas relaciones, resultado de los procesos y del kludge que emerge de ellos, lo que debería preocuparnos particularmente, antes que los procesos que serian tan solo indicadores de la dirección y potencial de los desarrollos que cada in- dustria emprende. El tejido de relaciones entre personas y actores sociales y económicos ilustra mejor lo que las decisiones interesadas de los actores transnacionales crean al desarrollar nuevos productos o servicios.

Para el Perú, la pregunta sobre la convergencia debería ser tanto qué hace la gente, cómo se relaciona con el nuevo entorno y cómo modifica su viejo entorno, a través de las relaciones socio técnicas creadas o potenciadas por las innovaciones; pero también habría que tomar en cuenta las prácticas mismas como el contexto o tejido que permite 
el surgimiento de esas relaciones entre personas y entre personas y actores sociales y económicos. Solo así la convergencia tendría sentido como fenómeno comunicacional. En otras palabras, ¿la convergencia fortalece o debilita los vínculos sociales y culturales? ¿Cómo lo hace?

Desde esta perspectiva, es posible pensar la convergencia de manera integrada pero al mismo tiempo, fragmentada: lo primero es una mirada conceptual, lo segundo una constatación de fenómenos que para los consumidores, son independientes. Por ejemplo, una familia puede adoptar una serie de kludges convergentes de distintos proveedores de servicios, creando una serie de relaciones entre consumidores y contenido, o prácticas de comunicación, que cambian las formas tradicionales de uso pero también las expectativas de usos y gratificaciones. Nuestra hipotética familia se suscribe a un paquete de servicios integrados (voz, tv de pago, Internet, quizá móviles, quizá portadores de larga distancia), lo que crea incentivos para alejarse de la provisión local de contenido y para fragmentar el consumo al interior de la familia, creándose nuevas relaciones sociales entre personas que consumen contenidos comparables o similares, es decir convergencia cultural. Pero los patrones de convergencia cultural estarán influenciados por las expectativas previas de satisfacción de necesidades culturales tanto como por la posibilidad de emular o copiar patrones de personas con las que se interac- túa cotidianamente, y por el descubrimiento individual de aquello que satisface intereses personales.

Bajo esta interpretación, la situación se convierte en una serie de pequeños campos de batalla individuales y específicos, antes que en una gran ola de renovación que sobrepasa las resistencias colectivas, como suele presentarse la convergencia. La provisión local, adosada como lo está a condiciones e intereses locales, podría ser una competencia significativa a los medios convergentes, siempre que los consumidores consideren que el contenido les es relevante; pero al mismo tiempo la atracción de los dispositivos convergentes personales como los teléfonos móviles (smartphones) lleva a buscar cómo darles sentido, especialmente cuando los usamos como extensiones "always-on, always-on-me", como las llama de forma intraducible Turkle (2008) (ver imagen 2).

De esto queda claro que lo más importante debería ser la salud de la oferta local, sin importar el medio; al mismo tiempo, la viabilidad económica de los negocios digitales es una cuestión todavía en debate, lo que hace necesario discutir la relación entre los medios masivos, analógicos o digitales, gratuitos o de pago; y los medios post-masivos, como los basados en la Internet. Mientras que los medios masivos de acceso libre al menos a nivel de propiedad y cobertura siguen siendo fundamentalmente nacionales, ya hace tiempo que el dominio del grueso de la 
Imagen 2. Los campos de batalla

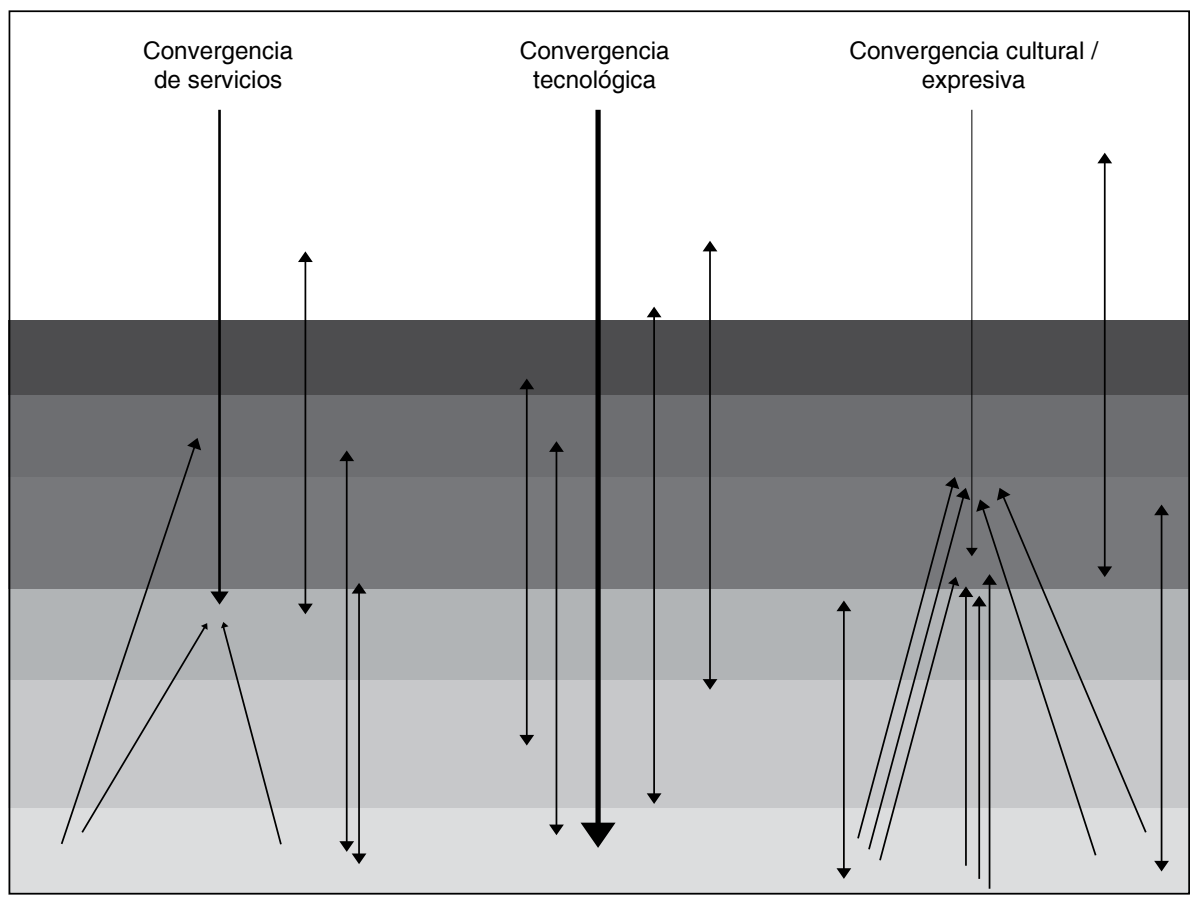

La oferta de contenidos local está en negro; las relaciones (flechas dobles) son los resultados hipotéticos del consumo convergente. Elaboración propia.

programación de medios masivos de pago recae en conglomerados sino globales, al menos transnacionales, y con segmentaciones tan claras que refuerzan la existencia de dos mercados peruanos: el limeño / cosmopolita y el específicamente local fuera de Lima y para los sectores de menores ingresos.

La inexistencia de un eco sistema que sostenga la producción nacional hace entonces que todos los avances tecnológicos o de servicios no generen relaciones entre los consumidores locales y los creadores de contenido locales, sino en segmentos altamente específicos; o que la creación local sea realmente local, limitada a los espacios de difusión de alcance local. Ya ha pasado, en buena medida, en la radio: las estaciones de alcance nacional ignoran buena parte de la producción local para abastecerse de contenidos estandarizados, que son difundidos por todo el país; son las estaciones locales las que pueden intentar resistir estas andanadas, que no son necesariamente cuestiones de convergencia sino en 
el sentido general de concentración de medios promovida por las relaciones emergentes. Esas muchas radios que repiten siempre las mismas viejas canciones, emitiendo su señal desde Lima pero para todo el país, son precisamente, "kludges", soluciones de corto plazo y de constitución improvisada alrededor de una necesidad específica: el sostenimiento comercial de una empresa. Los efectos socioculturales no están en el radar.

Ciertamente, la radio es un medio más bien estático, estancado por necesidad en una tecnología que no parece tener mayor posibilidad de cambio. Pero en la televisión la situación es diferente, bajo un supuesto proceso de cambio a cargo de la transición a la televisión digital terrestre (TDT). El "apagón analógico", que tendría que ser una ruta hacia la convergencia para las empresas de radiodifusión, ha sido pospuesto por la falta de adopción de receptores digitales en Lima, por lo menos hasta 2024 (Cassano, Dettleff \& Vásquez, 2015: 391), y muchas áreas del país no tienen ni siquiera una fecha de referencia para su propio apagón, con lo que el futuro de la TDT aparece como difuso sino dudoso, y con él, las posibilidades de consolidación empresarial más allá de los conglomerados limeños que controlan la transmisión de alcance nacional.

Entonces, en un intento de generalización, se puede plantear que en los países que cuentan simultáneamente con una industria de medios de comunica- ción nacional débil, excesivamente consolidada o sin alianzas con la industria de telecomunicaciones; al mismo tiempo que cuenta con una economía abierta y presencia significativa de inversiones y operaciones de transnacionales de telecomunicaciones y de informática, la convergencia significará que los actores dominantes locales corren el riesgo de ser aplastados por los servicios convergentes, no porque estos compitan directamente con ellos en los mismos mercados, sino porque los consumidores abandonarán los mercados de medios masivos en beneficio de los servicios convergentes. Los efectos de red producidos por los actores transnacionales empujarán a los actores locales a dedicarse a sectores de menores ingresos, lo que en casos como el peruano, significaría que los mercados se partirían en dos, con disminución y quizá desaparición de la presencia de los creadores culturales de la esfera mediática local, tal como ha ocurrido en la provisión tecnológica, y poco a poco en la provisión de servicios avanzados.

Recogiendo la propuesta de interpretación planteada al inicio de este artículo, estamos ante un conjunto de opciones que subsumen anteriores tecnologías, servicios y prácticas; el problema es que al hacerlo, reforzarían la tensión entre un sector de la población, cuya vocación cosmopolita se expresa en su interés por la oferta transnacional; y un sector fragmentado de consumidores orientados a lo local, que tienen que esperar por la llegada de contenidos 
convergentes aunque las industrias que los abastecen regularmente no llegan a crecer a la altura de las demandas del nuevo entorno.

\section{Perspectivas para investigación futura}

Con el incremento constante de la penetración de la Internet, cada vez más canales de TDT que sin embargo no logran aumentar la audiencia, medios con una estructura de propiedad altamente concentrada que difícilmente será tocada por el sistema político; los medios peruanos se muestran más bien débiles ante las presiones convergentes que ingresan en un mercado relativamente pequeño. La dualidad del mercado de medios hace particularmente complicada la subsistencia de industrias culturales dirigidas a toda la población, no solo a los sectores de mayores ingresos. La Internet seguirá subsumiendo en sí las tecnologías y los servicios, pero ¿qué pasará con la expresión de las mayorías?

Una ruta posible es la alianza entre las industrias mediáticas peruanas, con los conglomerados trasnacionales, para resistir los embates del contenido global o para darle formas nacionalmente reconocibles. Sin embargo el peso de estas firmas es mucho mayor, y la capacidad de negociación completamente inclinada a su propio interés. Además, el proceso de consolidación de los Unicornios no termina aún y es posible que algunos fracasos considerables ocurran en el futuro mediato, arrastrando con ellos a los aliados locales.
Por otro lado, las industrias locales en un país como el Perú deberían ser capaces de generar sus propios efectos de red, optimizando el acceso a tecnología y servicios para negociar en mejores condiciones con los operadores globales o con el público consumidor local y nacional. Sin estas negociaciones, solo un operador público podría resistir la influencia de lo global sin colapsar.

Todo esto indica que no hay reglas fijas para la convergencia; pero sin embargo es posible postular que hay una agenda académica, especialmente desde las comunicaciones, que puede servir para despejar las dudas. El estudio de la convergencia en el Perú, y en economías similarmente débiles en el campo de la comunicación, debería considerar:

- Cómo la convergencia es impulsada por los imperativos financieros de las empresas globales, bajo los cuales se decide la implementación local de ciertas tecnologías, servicios o contenidos. Esta faceta define el nivel de embate que los actores nacionales tendrán que enfrentar, y qué tecnologías y servicios podrían subsumir los actualmente existentes en cada país.

- Cómo los servicios convergentes se despliegan localmente, con qué nivel de autonomía se toman decisiones y con qué socios locales, nacionales o regionales se toman; para estimar el grado de resiliencia que los actores nacionales puedan tener a los embates ya mencionados. 
- Cómo y por qué se consume lo local, lo nacional y lo global, para conocer los efectos de la convergencia cultural en nuestras sociedades, lo que será la única forma real de estimar el peso de la subsunción de las distintas formas de expresión cultural en los patrones globales (esto también es válido para las prácticas profesionales), pero sobre todo nos ayudará a entender cuánta capacidad de resistencia tiene la sociedad en cuestión frente a las influencias de la cultura de consumo global, y cuánto logran los creadores locales y nacionales apoyarse en estos factores para seguir siendo relevantes para sus compatriotas.

Sobre todo, los estudios sociales y de comunicación han de insistir en que los beneficios de la convergencia, incluso si fueran tales como los que pro- mueven las empresas globales de telecomunicaciones y servicios, tienen que ser balanceados contra las realidades de desigualdades de ingreso; de desigualdad de acceso a los servicios y plataformas más activas y novedosas por parte de los creadores intelectuales y artísticos nacionales; y por el debilitamiento del espacio público que puede producirse por la fragmentación mediática. La consideración del rol de los estados, que no deben ignorar los efectos socioculturales de decisiones que parecen ser meramente técnicas o económicas, permitirá aportar en el debate sobre políticas públicas. Solo así se tendrá una mirada ponderada y finalmente, justa, sobre los cambios que la convergencia traerá; cambios que no por inevitables tienen que ser deseables en todos los casos.

\section{Referencias Bibliográficas}

Averweg, U.R. y Villanueva-Mansilla, E. (2009). Peruvian Cabinas Públicas: Does Policy Provide Practice or Does Practice Produce Policy? Information Technology in Developing Countries, 19(2), 9.

Beugnet, M. (2013). Miniature Pleasures: On Watching Films on an iPhone. En: Geiger, J., Littau, K., Cinematicity in Media History (196-200), Edinburgh: the University Press. doi:10.3366/edinburgh/9780748676118.003.0012.

Becerra, M. (2015). De la concentración a la convergencia: políticas de medios en Argentina y América Latina. Buenos Aires: Paidós.

Bustamante, E., Luna Victoria, J. (2014). El cine regional en el Perú. Contratexto, 22, $189-212$.

Campbell, J.E. (2011). It Takes an iVillage: Gender, Labor, and Community in the Age of Television-Internet Convergence. International Journal of Communication, 5, 492-510. 
Cassano, G., Dettleff, J., Vasquez Fermi, G. (2015). Perú: la ficción cede terreno. En: Orozco Gomez, G., Vasallo de Lopes, M. I. (coord.), Relaciones de género en la ficción televisiva: anuario Obitel 2015 (385-420), Porto Alegre: Sulina,

CEPAL. (2015). La nueva revolución digital: de la Internet del consumo a la Internet de la producción. Santiago: CEPAL. Disponible en http://www.cepal.org/es/ publicaciones/38604-la-nueva-revolucion-digital-la-internet-consumo-la-internetla-produccion

Cooke, L. (2005). A visual convergence of print, television, and the internet: charting 40 years of design change in news presentation. New Media and Society, 7(1), 22-46. DOI: $10.1177 / 1461444805049141$.

Crandall, R.W., Waverman, L. (1995). Talk is cheap: the promise of regulatory reform in North American telecommunications. Washington: Brookings Institution.

Dettleff, J. (2012). Las televisoras locales en el Perú: una historia de uso como herramienta política, el caso de Juliaca. Folios, 27, 161-184. Universidad de Antioquía Disponible en http://aprendeenlinea.udea.edu.co/revistas/index.php/folios/article/ viewFile/12772/11712.

Farber, D., Baran, P. (1977). The convergence of computing and telecommunications. En Cawkell, A. E. (1987). Evolution of an information society. London: Aslib, 230-239.

Finn, T.A., (1999). The role of temporality mediated communication and technology convergence. Information, Communication $\&$ Society, 2 (2), 174-200. DOI: $10.1080 / 136911899359691$

Gallegos, E. (2015). Cineastas del mundo andino: películas que son sensación en la sierra central. Diario Ojo, octubre 20, 2015.

Garnham, N. (1996). Constraints on multimedia convergence. En Dutton, W. H. (ed.). Information and communication technologies: visions and realities. Oxford: OUP, 103-120.

Gutiérrez-Rentería, M. E., Lopez, C. E. (2014). La convergencia digital propicia convergencia entre industrias: principales empresas que participan en el mercado de capitales, concentran los mayores ingresos en 2013. Revista de Comunicación, 144-162. Disponible en http://udep.edu.pe/comunicacion/rcom/es/articulos/2014/ Art144-162.html

Harvard. (2016). How unicorns grow. Harvard Business Review: Entrepreneurial finance. Disponible en https://hbr.org/2016/01/how-unicorns-grow

Hier, S. (2008). Transformative democracy in the age of second modernity: cosmopolitanization, communicative agency and the reflexive subject. New Media and 
Society, 10(1) 27-44. DOI: 10.1177/1461444807085320.

Huamán, F., Becerra, C. (2014). Debate sobre la concentración de medios en el Perú: el caso de la fusión del grupo El Comercio con el grupo EPENSA. Ponencia presentada al XII Congreso de ALAIC, agosto 2014. Disponible en http://congreso.pucp.edu.pe/ alaic2014/wp-content/uploads/2013/11/vGT18-Huaman-Becerra.pdf

Humphreys, L., Von Pape, T. y Karnowski, V. (2013). Evolving Mobile Media: Uses and Conceptualizations of the Mobile Internet. Journal of Computer-Mediated Communication, 18, 491-507. DOI: 10.1111/jcc4.12019.

Ishii, K. (2006). Implications of mobility: the uses of personal communication media in everyday life. Journal of Communication, 56 (2), 346-356.

Jenkins, H. (2006). Convergence culture: where old and new media collide. Nueva York: NYU Press.

Jenkins, H., Ford, S. y Green, J. (2013). Spreadable media: creating value and meaning in a networked culture. Nueva York: NYU Press.

Lim, J. (2010). Convergence of Attention and Prominence Dimensions of Salience among Major Online Newspapers. Journal of Computer-Mediated Communication, 15, 293-313. doi:10.1111/j.1083-6101.2010.01521.x

Lin, C.C. (2013). Convergence of new and old media: new media representation in traditional news. Chinese Journal of Communication, 6 (2), 183-201. DOI: $10.1080 / 17544750.2013 .785667$

MacKenzie, A. (2010). Wirelessness: radical empiricism in network cultures. Cambridge, MA: MIT.

Mansell, R. (1993). The new telecommunications: a political economy of network evolution. London: Sage.

Mastrini, G., Bolaño, C. (eds.). (1999). Globalización y monopolios en la comunicación en América Latina: hacia una economía política de la comunicación. Buenos Aires: Biblos.

McChesney, R. (1999). Rich media, poor democracy: communication politics in dubious times. New York: The New Press.

McPherson, T. (2008). "The End of TV As We Know It": Convergence Anxiety, Generic Innovation, and the Case of 24. En Kolker, R. (ed.). The Oxford Handbook of Film and Media Studies (306-319). Oxford: OUP,

Melody W. (2014). Whose global village? En: Mansell, R. y Raboy, M. The handbook of global media and communication policy (58-78), Oxford: Wiley-Blackwell.

Mendoza, M. (2015). Gestión de las empresas periodísticas regionales: El Sol, del Cusco; El Tiempo, de Piura e Impetu, de Ucayali. Revista de Comunicación, 
14, 70-99. Disponible en http://udep.edu.pe/comunicacion/rcom/pdf/2015/ Art070-099.pdf

Mitchelstein, E. y Boczkowski, P. J. (2013). Tradition and Transformation in Online News Production and Consumption. En: Dutton, W. (ed.). The Oxford Handbook of Internet Studies, (78-97). Oxford: OUP.

Morales, R., Solórzano, R., Távara, J.I., Villanueva-Mansilla, E. (2009). Contribución Econoómica de las Industrias Basadas en Derechos de Autor en el Perú. Preparado para la Organización Mundial de la Propiedad Intelectual (OMPI) y el Instituto Nacional de la Competencia y de la Protección de la Propiedad Intelectual (INDECOPI). Lima. https:/www.indecopi.gob.pe/documents/20182/143803/ ContribucionEconomicaIndustriasDAPeru.pdf

Neuchterlein, J.E. y Weiser, P.J. (2005). Digital crossroads: American telecommunications policy in the Internet age. Cambridge, MA: MIT.

Peru-MTC (Ministry of Transportation and Communications). (2011). Plan Nacional para el desarrollo de la banda ancha en el Perú. Lima: MTC.

Robinson, S. (2006). Journalism and the Internet (review article). New Media and Society, 8 (5), 843-849. DOI: 10.1177/1461444806067592.

Salaverría, R. (2008). Periodismo integrado: convergencia de medios y reorganización de redacciones. Barcelona: Editorial Sol90.

Santander, P. (2014). Nuevas leyes de medios en Sudamérica: enfrentando políticamente la concentración mediática. Convergencia, 21(66). Disponible en http://www. scielo.org.mx/scielo.php?script =sci_arttext\&pid $=$ S1405-14352014000300001

Scolari, C. (2015). From (new) media to (hyper) mediations: Recovering Jesús Martín-Barbero's mediation theory in the age of digital communication and cultural convergence. Information, Communication and Society, 18(9), 1092-1107. DOI: 10.1080/1369118X.2015.1018299.

Scolari, C.A., Ibrus, I. (2014). Transmedia Critical: Empirical Investigations into Multiplatform and Collaborative Storytelling Introduction. International Journal of Communication, 8, 2191-2200.

Tambini, D., Leonardi, D., Marsden, C. (2008). Codifying Cyberspace: Communications Self-Regulation in the Age of Internet Convergence. Nueva York: Routledge.

Tucker, J. (2013). Gentleman Troubadours and Andean Pop Stars: Huayno Music, Media Work, and Ethnic Imaginaries in Urban Peru. Chicago: the University Press.

Turkle, S. (2008). Always-on, always-on-you: the tethered self. En: Katz, J. (ed.), Handbook of Mobile communication studies (121-138). Cambridge: MIT. 
Valtysson, B. (2012). Regulating Convergence. Information, Communication छ Society, 15(6), 982-983. DOI: 10.1080/1369118X.2011.595815.

Russell, A., Ito, M., Richmond, T., Tutters, M. (2008). Culture: media convergence and networked participation. En: Varnelis, K. (ed). Networked publics, (43-61). Cambridge: MIT.

Villanueva-Mansilla, E. (2000). Convergencia multimedia: más allá de la Internet. Diálogos de la comunicación, 59-60, 225-232.

Villanueva-Mansilla, E., Nakano, T. y Evaristo, I. (2015). From divides to capitals: an exploration of digital divides as expressions of social and cultural capital. Communication and Information Technologies Annual (89-117). Londres: Emerald.

Webster, J.G., Ksiazek, T.B. (2012). The Dynamics of Audience Fragmentation: Public Attention in an Age of Digital Media. Journal of Communication, 62 (1), 39-56. doi:10.1111/j.1460-2466.2011.01616.x.

Weinstein, S. (2004). OFCOM, information-convergence and the never-ending drizzle of electric rain. International Journal of Communications Law and Policy, 8, $1-39$.

Yuan, E. (2011). News consumption across multiple media platforms. Information, Communication and Society, 14 (7), 998-1016. DOI: 10.1080/1369118X.2010.549235 